\title{
A Numerical Analysis on a Single Bunch of Wires of Susstain-El: The First Italian Elastocaloric Device
}

\author{
Luca Cirillo, Adriana Greco, Claudia Masselli*
}

DII, University of Naples Federico II, Pl.e Tecchio 80, Naples 80125, Italy

Corresponding Author Email: claudia.masselli@unina.it

https://doi.org/10.18280/ijht.390601

Received: 24 September 2021

Accepted: 13 December 2021

\section{Keywords:}

elastocaloric cooling, active elastocaloric refrigeration system, SMA wires, 2-D Model

\begin{abstract}
Refrigeration is responsible of twenty percent of the worldwide energy consumption and the majority of the systems are based on vapor compression.

In the scientific community, elastocaloric refrigeration, belonging to solid-state refrigeration technologies, is increasingly attracting interest, as a valid alternative to vapour compression. It is based on the latent heat generated as a consequence of the austenitemartensite transformation phase, a phenomenon shown by Shape Memory Alloys (SMA) as a consequence of cycles of stress/unstress under adiabatic conditions. The main SMA property is the capability of keeping memory of the initial form and being able to recover it after the remotion of the uniaxial stress (unloading). The elastocaloric effect can be exploited in a regenerative thermodynamic cycle called Active elastocaloric regenerative refrigeration cycle (AeR). The paper reports the data coming out though a numerical analysis by modelling a single bunch of elastocaloric wires crossed by air. The heat transfer and the energy performances have been investigated under a wide set of conditions: different geometrical parameters of the wires and many air flow speed.
\end{abstract}

\section{INTRODUCTION}

The research for novel technologies for cooling and air conditioning has been pushed by the new directives prescribing [1] a progressive dismission of refrigerants fluids because of their impact on global warming and atmosphere caused by high Global Warming Potential (GWP) and not-zero Ozone Depletion Potential (ODP). Over the years more space has been gained by unconventional refrigeration, known as Not-in-Kind [2-4], basing on solid-state materials showing caloric effect [5]. The latter is a phenomenon where solid-state refrigerants exhibit a temperature variation as a consequence of an external stimulus coming from an adiabatic variation of an external field [6]. These materials do not present any GWP that consequentially derives in a more ecological impact of caloric technology toward the environment, rather than vapor compression [7-9]. The caloric technologies include magnetocaloric, electrocaloric, elastocaloric and barocaloric materials [10]; depending on the type of stimulus given by the external field. Magnetocaloric effect rises as a consequence of the application of a magnetic field $[11,12]$; electrocaloric one is forced by the variation of an electric field [13]; elastocaloric is the results of a mechanical stress [14] and barocaloric effect $[15,16]$ depends on the application of a hydro-static pressure.

The primary of the above mentioned technologies developed and investigated over the last 30 years is the magnetocaloric one [17] through manly the employment of Gadolinium, is the benchmark magnetocaloric material that exhibits a peak of the effect at room temperature (294 K). The results of the investigation in this fields are clearly visible since currently more than 100 prototypes of magnetocaloric refrigerators or heat pump were developed [18], but the goal of the commercialization was not scored, yet. The reasons are attributable to the small values of Coefficients of Performance (COPs), cooling power and temperature span that a magnetocaloric refrigerator could achieve, since limited are the $\Delta \mathrm{T}_{\mathrm{ad}}$ detected in the magnetocaloric materials. Moreover, many of them are not easily available for a large scale use (rare earths) and low are the intensities of the magnetic fields that a permanent magnet could generate.

The natural and more rational consequence of the above conclusions has been a wider extension of the researches toward the other caloric effect-based techniques. Elastocaloric cooling seems to be the most encouraging since the materials are cheap, easy to produce as well as simpler is the application of the mechanical stimulus. The elastocaloric benchmark material is the Ni-Ti binary alloy $[19,20]$, that are Shape Memory Alloys (SMA) showing elastocaloric effect at ambient temperature. These materials are capable to memorize the shape they have before being uniaxially stressed and the possibility to come back in that shape once the mechanical field was removed. When the stress is applied (loading process), the elastocaloric material transforms itself structurally from Austenite to Martensite (A-M); whereas a MA transformation takes place when the stress is removed (unloading process). The shape-memory and the super elasticity, exhibited aabove austenite stability temperature $\left(\mathrm{A}_{\mathrm{f}}\right)$ and below martensite stability temperature $\left(\mathrm{M}_{\mathrm{f}}\right)$, respectively, are two properties of the elastocaloric materials strictly connected to A-M and $\mathrm{M}-\mathrm{A}$ transformation. The latter property allows the SMA being loaded up to $8.5 \%$ strain, thus releasing the transformation heat; on the other side, the former property allows to recover the initial shape, avoiding deformations.

The correct and customized developing with respect to the desired purposes, depends crucially on the elastocaloric 
materials employed. Since the first pioneering investigations, the NiTi binary alloys got unquestionably the benchmark role, caused by the promising temperature changes shown at room temperature. The latter are classifiable as Shape Memory Alloys but elastocaloric materials can be alternatively Shape Memory Polymers (SMP).

Shape Memory Alloys are all the Ni-Ti based alloys (doped with $\mathrm{Cu}, \mathrm{Co}$, Pd, etc.), the Cu-based (doped with $\mathrm{Al}, \mathrm{Ni}, \mathrm{Zn}$, $\mathrm{Mn}$, etc.) and Fe-based based (doped with Pd, Rh, Mn, Si, Ni, etc.) binary ternary or quaternary alloys [21]. Recent investigations revealed that the combination of a Nickel Titanium alloy with vanadium or aluminum minimizes the stress hysteresis and stretch the fatigue life [22]. The addition of copper as fourth element of the alloy makes the A-M/M-A transformations tunable in the desired temperature range [23, 24]. Other researchers noticed that the presence of aluminum as third element of a $\mathrm{Cu}$-based alloy (Cu-Zn-Al [25], Cu-Al$\mathrm{Mn}$ [26]) improves the conductivity of the elastocaloric element and thus the heat transfer coefficients. The latent heat released during the entire A-M adiabatic transformation in the $\mathrm{Cu}-\mathrm{Zn}-\mathrm{Al}$ alloys provokes a $15 \mathrm{~K}$ temperature increase; the con is the short fatigue life of such ternary elastocaloric materials that condition the application requiring long-time working. In the end, Fe-based alloys exhibit temperature changes significantly lower than the other two families $(5 \mathrm{~K})$ but smaller is the stress hysteresis, too [27].

Rubbers and polymers are the classes of elastocaloric materials defined SMP $[28,29]$ and they are cheaper than $\mathrm{Ni}-$ Ti alloys and due of a longer fatigue life but enormous strains (up to $600 \%$ ) are required to observe the elastocaloric effect, i.e. the coupled significant elongations yield SMP not to easily employable for practical applications in experimental prototypes.

The keys to develop a well-operating elastocaloric experimental protype is not limited to the choice of the most appropriate material but an optimized projecting is required, and this aspect can preliminary being studied through numerical modelling.

Currently, the elastocaloric devices developed and introduced to the caloric scientific community are no more than 15 and far is the possibly of large-scale employment, yet.

Italy has not presented yet any developed prototype to the worldwide community but the University of Naples Federico II is going to fill this gap through the "SUSSTAINEBLE" research project $[30,31]$. The final goal of SUSSTAINEBLE is to develop a the first Italian elastocaloric prototype featured by the rotation of several bunches of elastocaloric wires crossed by air as heat transfer fluid. In this way the bunches experiment their Active elastocaloric Regenerative (AeR) cycle not synchronically each other. The four processes of the AeR cycle are:

(1) the application of the stress $\sigma$ to adiabatically load the SMA up to making the A-M transformation happens and observing a positive $\Delta \mathrm{T}_{\text {ad }}(\mathrm{T}, \sigma)$ in the SMA;

(2) a heat transfer is the consequence of the air (colder) flowing through the wires to absorb heat from them and release it the environment or in a hot heat exchanger;

(3) the unloading of the SMA by reducing the stress from $\sigma$ to 0 gives rise to M-A and a negative $\Delta \mathrm{T}_{\text {ad }}(\mathrm{T}, \sigma)$ is registered;

(4) the air (hotter) flowing through the SMA wires (colder) allows the former to cool down and producing the cooling effect, once reached the environment or the cold heat exchanger.

A numerical investigation has been performed in this paper to evaluate the energy performances of a single bunch of wires, following the variation of many parameters: cycle frequency, air velocity, wires length and distance among them.

\section{THE MATHEMATICAL MODEL}

A single bunch of NiTi wires has been modelled since it is one of the elements that act as solid-state refrigerant, that will be mounted on the ring of the rotary prototype operating continuously. Specifically, it is conceived as formed by several bunches of elastocaloric wires placed around the circumference of two co-rotating disks. The aim is to develop an elastocaloric refrigerator prototype as a direct air-cooling unit, without using additional heat exchangers, i.e. air crosses the SMA bunches during the rotation of the ring, by flowing in the channel and investing them parallelly. The continuous extraction of hot and cold air is allowed by an appropriate positioning of the air inlets and outlets on the prototype base. To optimize the geometrical parameters of the elastocaloric elements to be mounted in the prototype, a single bunch of wires was modelled. In this way it is also possible to evaluate the energy performances while many operating parameters are varying: rotation frequency, air velocity, wires length, distance between wires.

The material under test in the present investigation is the benchmark $\mathrm{Ni}_{50.4} \mathrm{Ti}_{49.6}$, with an Austenite finish temperature $\mathrm{A}_{\mathrm{f}}$ of $20^{\circ} \mathrm{C}$. This temperature allows to achieve the pseudo elastic behavior at ambient temperature. For this material, the complete Austenite-Martensite transformation corresponds to a latent heat of $20 \mathrm{~J} \mathrm{~g} \mathrm{~g}^{-1}$ and to an adiabatic temperature change of $40 \mathrm{~K}$. The SMA was subjected to a strain rate of $0.25 \mathrm{~s}^{-1}$ that corresponds to an adiabatic transformation with a loading and unloading time of around $200 \mathrm{~ms}$. We avoided to solicit the wires with larger strain rate because of the crucial consequential reduction of fatigue life. The time variation of the fluid flow has allowed to vary the operating frequency.

The supporting mathematical model is described by the following equations:

$$
\left\{\begin{array}{c}
\frac{\partial u}{\partial x}+\frac{\partial v}{\partial y}=0 \\
\frac{\partial u}{\partial t}+u \frac{\partial u}{\partial x}+v \frac{\partial u}{\partial y}= \\
-\frac{1}{\rho_{f}} \frac{\partial p}{\partial x}+v_{f}\left(\frac{\partial^{2} u}{\partial x^{2}}+\frac{\partial^{2} u}{\partial y^{2}}\right) \\
\frac{\partial v}{\partial t}+u \frac{\partial v}{\partial x}+v \frac{\partial v}{\partial y}= \\
-\frac{1}{\rho_{f}} \frac{\partial p}{\partial y}+v_{f}\left(\frac{\partial^{2} v}{\partial x^{2}}+\frac{\partial^{2} v}{\partial y^{2}}\right) \\
\rho_{f} c_{f} \frac{\partial T_{f}}{\partial t}+\rho_{f} c_{f}\left(u \frac{\partial T_{f}}{\partial x}+v \frac{\partial T_{f}}{\partial y}\right) \\
=k_{f}\left(\frac{\partial^{2} T_{f}}{\partial x^{2}}+\frac{\partial^{2} T_{f}}{\partial y^{2}}\right) \\
\rho_{S M A} c_{S M A} \frac{\partial T_{S M A}}{\partial t} \\
=k_{S M A}\left(\frac{\partial^{2} T_{S M A}}{\partial x^{2}}+\frac{\partial^{2} T_{S M A}}{\partial y^{2}}\right)+\mathrm{G}
\end{array}\right.
$$

To develop the model, the following assumption have been made: i) negligible is the loading/unloading time with respect 
to the heat transfer time caused by the air flow. This ensure the loading/unloading process to be considered adiabatic. ii) the A-M and M-A transformations occurs uniformly though uniaxially stretching operations; iii) the external boundary conditions of the regenerator ensure it is adiabatic with respect to the environment; iv) the model is 2-dimensional; v) the elastocaloric effect is evaluated through the estimation of the latent heat and the work needed for loading/unloading processes.

where, $\mathrm{G}$ is the heat source due to elastocaloric effect, i.e. it depends on the latent heat $\Delta H$, the martensitic phase transformation $\dot{\xi}_{M}$ and the work required $w$ and it can be evaluated as:

$$
G=\rho_{S M A}(\Delta H+w) \dot{\xi}_{M}
$$

In SMA elements the heat source associated to the A-M and M-A transformations is different due to the stress hysteresis. According to Tusek et al. [32] the net-work is:

$$
w_{\text {net }}=w_{\text {load }}-w_{\text {unload }}
$$

representing the entropy-temperature hysteresis cycle area

The martensitic phase transformation $\dot{\xi}_{M}$ can be estimated through the following differential Eq. (4):

$$
\dot{\xi}_{M}=\xi_{M} \psi^{M A}\left(T_{S M A}, \sigma\right)-\xi_{A} \psi^{A M}\left(T_{S M A}, \sigma\right)
$$

where:

$$
\xi_{M}+\xi_{A}=1
$$

Since the time required for the loading/unloading processes is negligible compared to the heat exchange time with air, the term $\dot{\xi}_{M}$ assumes only +1 or -1 as numeric value. Therefore, $G$ changes its sign during the A-M/M-A transformations. During the convective heat transfer process, the elastocaloric material is kept in a fixed strain condition, thus $\dot{\xi}_{M}=0$ and consequently the heat source term $\mathrm{G}$ is zero.

\section{OPERATING CONDITIONS}

The air flow crossing the elastocaloric wires has an entering temperature of $293 \mathrm{~K}$ on both sides since in this preliminary investigation we do not connect the cold and hot heat exchangers to the device, and we suppose direct exchanges with the environment where the prototype is placed. $0.25 \mathrm{~s}^{-1}$ is the strain rate for the stress/unstress cycles on Ni-Ti wires, thus corresponding to a time period of 0.2 each. Keeping constant this time, the working frequency of the AeR cycle was changed depending on the time for fluid flowing. The thermophysical properties of $\mathrm{Ni}_{50.4} \mathrm{Ti}_{49.6}$ are: $15 \mathrm{~W} \mathrm{~m}^{-1} \mathrm{~K}^{-1}$ as thermal conductivity; $6500 \mathrm{~kg} \mathrm{~m}^{-3}$ as density; $500 \mathrm{~J} \mathrm{~kg}^{-1} \mathrm{~K}^{-1}$ as specific heat [33].

The cycle frequency, i.e. the time for fluid flowing was set in relation to the transient constant $\tau$ that regulates the convective heat exchange between the fluid and the elastocaloric material:

$$
\tau=\frac{\rho_{S M A} c_{S M A} V_{\text {wire }}}{h A_{\text {wire }}}=\frac{\rho_{S M A} c_{S M A} D_{\text {wire }}}{4 h}
$$

The (6) underlines that the smaller the wire diameter is, the higher will be the cycle frequency. In these simulations the wires are designed with a diameter of $0.3 \mathrm{~mm}$, since a smaller value would have carried the elastocaloric elements to break with greater ease.

The set of the values of the time for fluid flow used in the investigation (always multiples of $\mathrm{t}$ ) is: $t_{f}=$ $[1.0 ; 2.0 ; 4.0 ; 6.0] s$.

The set of the air velocity, chosen according to the typical values of the air-cooling systems, is: $\vec{v}=$ $[3.0 ; 5.0 ; 7.0 ; 9.0 ; 11.0] \mathrm{m} \mathrm{s}^{-1}$.

The elastocaloric elements have been designed placed at three different distances each other: $\boldsymbol{d}=[0.5 ; 1 ; 2] \mathrm{mm}$.

Variable is also the wires length: $\boldsymbol{L}=[100 ; 200 ; 300] \mathrm{mm}$.

After each investigation the results have been collected and studied through the evaluation of the main energy performance indexes of an air-conditions: the temperature span and the AeR cooling power, defined respectively in Eq. (7) and (8), were calculated after each test:

$$
\Delta T_{\text {span }}=\frac{1}{\theta}\left(\begin{array}{c}
\int_{t_{\text {load }}+n \theta}^{t_{\text {load }}+t_{C H T}+n \theta} \\
\int_{f}(L, y, t) d t \\
\int_{t_{\text {load }}+t_{C H T}+t_{\text {unload }}+n \theta}^{t_{\text {load }}+2 t_{C H T}+t_{\text {unload }}+n \theta} \\
T_{f}(0, y, t) d t
\end{array}\right)
$$

The temperature span is defined as the difference between the average temperature of the hot air leaving the regenerator in the second step of the cycle (when the heat is released) and the temperature of the cold air exiting from the regenerator in the fourth step of the cycle.

$$
\begin{gathered}
\dot{Q}_{\text {ref }}=\frac{1}{\theta} \int_{t_{\text {load }}+t_{f}+t_{\text {unload }}+n \theta}^{t_{\text {load }}+2 * t_{f}+t_{\text {unload }}+n \theta} \dot{m}_{f} C_{f}\left(T_{\text {env }}\right. \\
\left.-T_{f}(0, y, t)\right) d t
\end{gathered}
$$

The cooling power is calculated as the integral in the heat exchange time of the difference between environmental temperature $(293 \mathrm{~K})$ and the temperature of the cold air exiting from the system in the last step of the cycle.

\section{RESULTS AND DISCUSSION}

The Figure 1 shows the temperature span vs the cycle frequency parametrized for air velocity when the distance between two wires is: (a) $0.5 \mathrm{~mm}$; (b) $1.0 \mathrm{~mm}$; (c) $2.0 \mathrm{~mm}$. It could be noticed that the smaller is the air flow speed, the lower is the frequency that maximizes the temperature span. The reason is to be found in the higher values of convective heat exchange transient constant corresponding to smaller velocity, since larger is the time for optimal heat exchange. At the same time, at fixed velocity, it could be noticed that the greater the distance between two wires is, the higher is the optimal frequency point.

At fixed distance between two wires and for every distance, the data is a reduction of the temperature span for larger air velocities; such data is more remarkable if smaller are the AeR cycle frequencies. $40 \%$ is the value of the mean decrement. To well understand the previously mentioned trend one can consider that if the air flows slowly, greater is the time that air 
spends in contact with the wires, i.e. major is the heat exchange, with a consequential augmentation of the heat transfer.

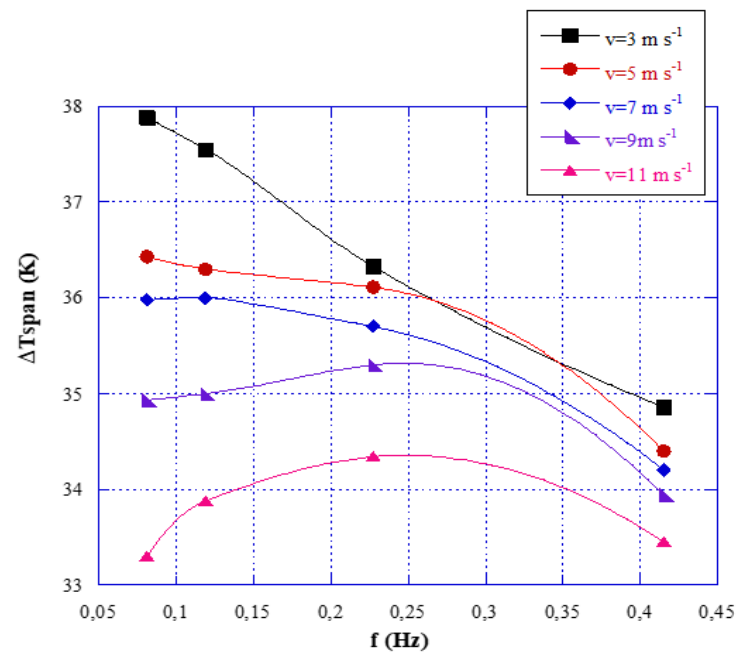

(a)

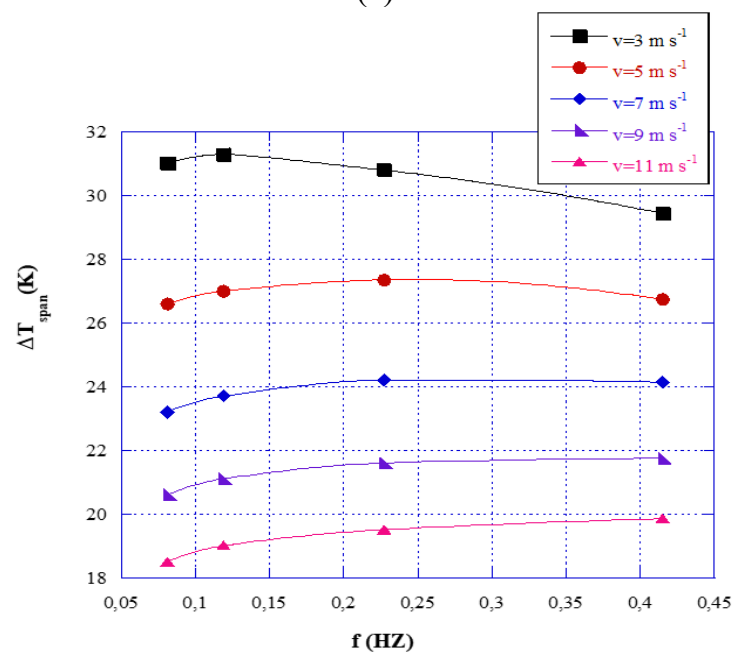

(b)

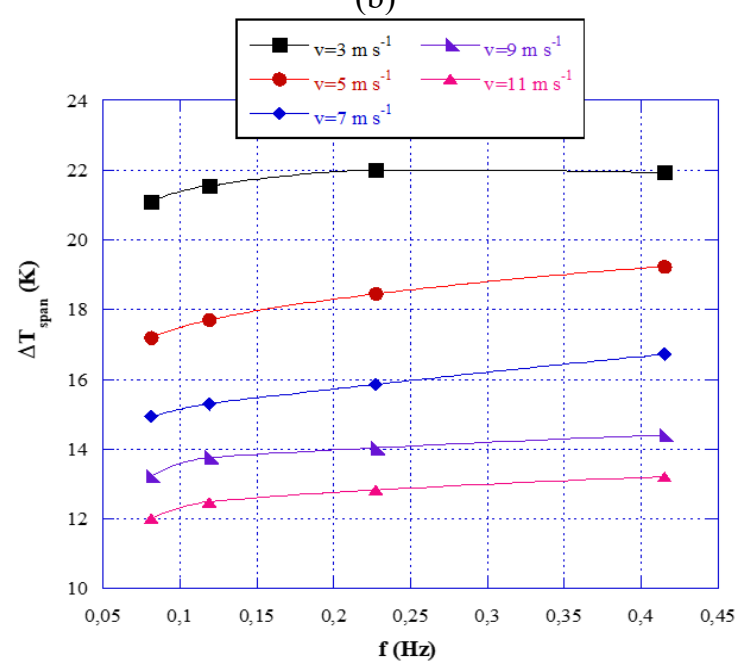

(c)

Figure 1. $\Delta \mathrm{T}_{\text {span }} \mathrm{vs} \mathrm{f}_{\mathrm{AeR}}$ parametrized for air velocity when the distance between two consecutive wires is: (a) $0.5 \mathrm{~mm}$; (b) $1.0 \mathrm{~mm}$; (c) $2.0 \mathrm{~mm}$

The placement of the wires at smaller distances each other carries to the detection of larger temperature span $(+50 \%$ as medium increment on the $\Delta \mathrm{T}_{\text {span }}$ values calculated for $\mathrm{d}=0.5$ $\mathrm{mm}$ with respect to $\mathrm{d}=1.0 \mathrm{~mm}$ ). The increment is larger if faster is the fluid flowing (the mean increment is $40 \%$ at 3 $\mathrm{m} \mathrm{s}^{-1}$ air velocity and $60 \%$ at $11 \mathrm{~m} \mathrm{~s}^{-1}$ ). Indeed, the placement of the wires at reduced distance each other provokes an increment of the convective heat transfer coefficient (on equal air flow speed), increasing the heat exchange between the fluid and the SMA elements.

The $\dot{Q}_{\text {Cool }}$ per mass unit of Ni-Ti as a function of frequency parametrized for fluid velocity when the distance between two wires is: (a) $0.5 \mathrm{~mm}$; (b) $1.0 \mathrm{~mm}$; (c) $2.0 \mathrm{~mm}$ is visible in Figure 2.

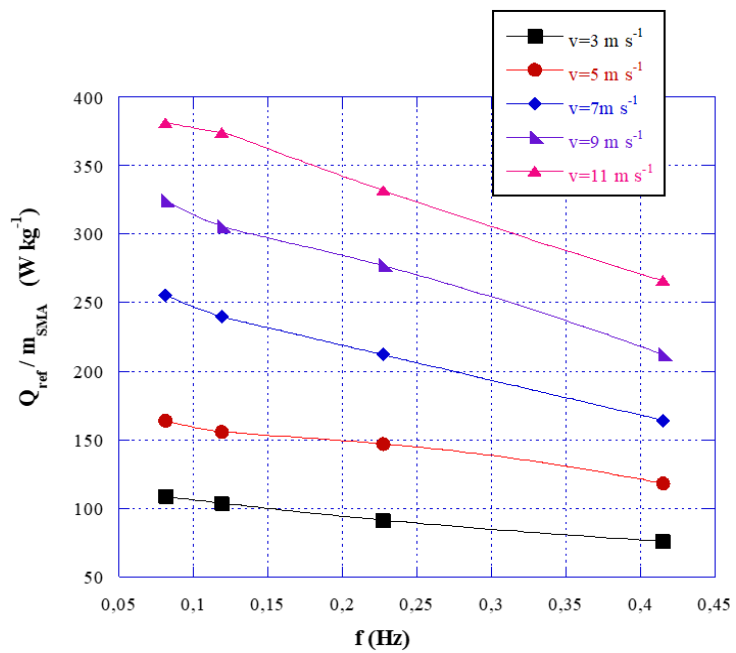

(a)

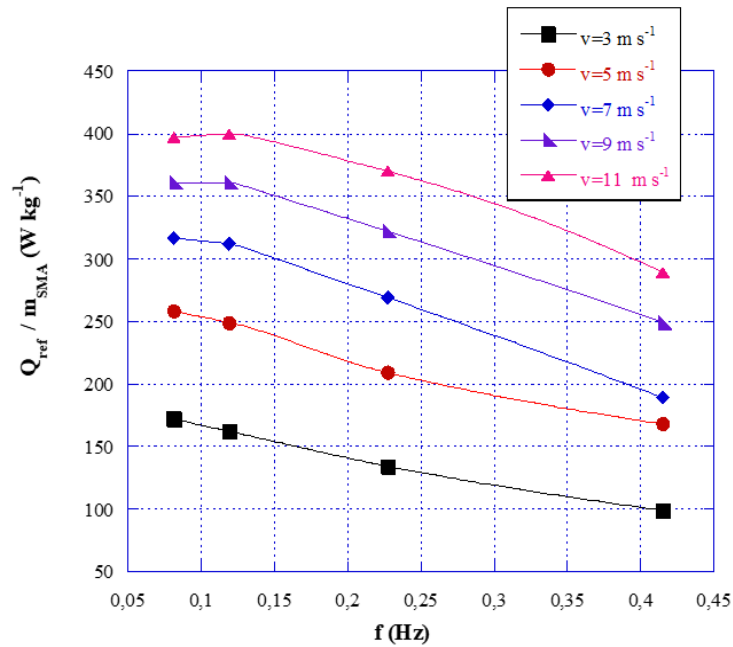

(b)

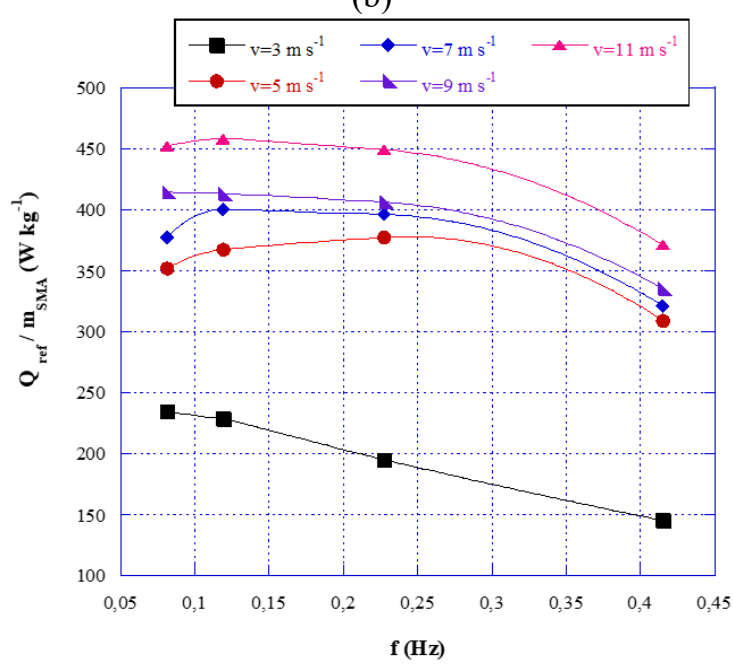

(c)

Figure 2. Cooling power per mass of the elastocaloric element mass vs $f_{A e R}$ parametrized for $v$ when $d$ is: (a) 0.5 $\mathrm{mm}$; (b) $1.0 \mathrm{~mm}$; (c) $2.0 \mathrm{~mm}$ 
The examinations of the curves plotted in the Figures 2 (a), (b), (c) reveals that for all wire distance d large cooling powers are associated to faster air velocities and the medium increment with speed increasing $+60 \%$, specifically between $3 \mathrm{~m} \mathrm{~s}^{-1}$ and $11 \mathrm{~m} \mathrm{~s}^{-1}$.

Indeed, on equal frequency for faster fluid flows greater heat transfer coefficients are calculated, thus improving the solid-fluid heat transfer. This increment is better visible is smaller is the distance between the wires $(+70 \%$ as medium increment at $\mathrm{d}=0.5 \mathrm{~mm}$ whereas $+50 \%$ is at $\mathrm{d}=2 \mathrm{~mm}$ ). The is an optimal frequency for every curve of iso-velocity. The maximum value of the $\dot{Q}_{C o o l}$ and the maximum value of the $\Delta \mathrm{T}_{\text {span }}$ correspond to different value of the optimal frequency.

The cooling power increases if the wires are placed farer due to the augmentation of the global fluid flow rate in the prototype (at fixed number of wires). It is higher when the air flow is lower. For slower air speeds, for example at $3 \mathrm{~m} \mathrm{~s}^{-1}$, the geometry configuration where the wires are placed at $1.0 \mathrm{~mm}$ or $2.0 \mathrm{~mm}$ as a distance, gives respectively $+50 \%$ and $+40 \%$ as medium cooling power per mass unit increase with respect to $\mathrm{d}=0.5 \mathrm{~mm}$; if the speed grows up to $11 \mathrm{~m} \mathrm{~s}^{-1}$, the registered increment is only $+8 \%$ and $+18 \%$, respectively for $1.0 \mathrm{~mm}$ and $2.0 \mathrm{~mm}$.

There is compromise between the achievement of the maximum of $\Delta \mathrm{T}_{\mathrm{span}}$ and the maximization of $\dot{Q}_{\mathrm{Cool}}$. For this reason, a middle resolution could be working with parameters set at the center of the range and choosing $1.00 \mathrm{~mm}$ as optimal distance between the SMA elements. Once the previous parameter was established, the wires length has been investigated. In the Figures $3 \Delta \mathrm{T}_{\text {span }}$ as a function of $f_{A e R}$ parametrized for air velocity when the length of the wires is: (a) $100 \mathrm{~mm}$; (b) $200 \mathrm{~mm}$; (c) $300 \mathrm{~mm}$, is reported. To smaller speeds higher values of temperature span are coupled. The increasing of wire length positively impacts on $\Delta \mathrm{T}_{\mathrm{span}}$, stemming anyhow that the relative percentage increments with the length are effectively function of the fluid velocity, more than cycle frequency. For $3 \mathrm{~m} \mathrm{~s}^{-1}$ speed, a medium value (with respect to the tested frequencies) of $+7 \%$ on $\Delta \mathrm{T}_{\text {span }}$ has been calculate if the length increases from $100 \mathrm{~mm}$ to $300 \mathrm{~mm}$, whereas $+69 \%$ is shown for $11 \mathrm{~m} \mathrm{~s}^{-1}$ air speed. The increase of the $\Delta \mathrm{T}_{\text {span }}$ if the lengths augments from $200 \mathrm{~mm}$ to $300 \mathrm{~mm}$ is detectable only for high speed whereas it is completely inexistent for $3 \mathrm{~m} \mathrm{~s}^{-1}$.

The differences between the absolute maximum and the absolute minimum, proper of each data set are: $12.7 \mathrm{~K}$ for $\mathrm{L}=100 \mathrm{~mm} ; 7.3 \mathrm{~K}$ for $\mathrm{L}=200 \mathrm{~mm} ; 4.4 \mathrm{~K}$ for $\mathrm{L}=300 \mathrm{~mm}$. From Figure 3 one can assert that at $100 \mathrm{~mm}$ there is the most sensitivity to the variation of the investigated working parameters: $\mathrm{v}$ and $\mathrm{f}_{\mathrm{AeR}}$.

The effect of the length variation has been studied also with respect to the cooling power for mass unit, as Figure 4 shows as a function of $\mathrm{f}_{\mathrm{AeR}}$ parametrized for $\mathrm{v}$ when the length of the wires is: (a) $100 \mathrm{~mm}$; (b) $200 \mathrm{~mm}$; (c) $300 \mathrm{~mm}$.

The general consideration emerging from Figure 4 is that higher cooling powers per mass unit are noticeable for larger air flow velocities and there is a different optimal frequency depending on the length. Furthermore, the $\dot{Q}_{\text {Cool }}$ per unit mass rises with the wire length: more precisely, increasing the wire length, the heat exchange paths that the air crosses are longer; indeed, there is an augmentation of the heat exchange area. When the length grows up the effect on $\dot{Q}_{\text {Cool }}$ is always very pronounced if $\mathrm{L}$ grows from $100 \mathrm{~mm}$ to $200 \mathrm{~mm}$, for all the air velocities and the frequencies tested. Anyhow if $\mathrm{L}$ increases from $200 \mathrm{~mm}$ to $300 \mathrm{~mm}$ a saturation in the cooling power augmentation is evident for all the frequencies at low speeds, above all $3 \mathrm{~m} \mathrm{~s}^{-1}$. Increasing the air velocity, the saturation effect is evident only from the frequency $0.227 \mathrm{~Hz}$ upwards.

At $3 \mathrm{~m} \mathrm{~s}^{-1}$, the effect of augmenting L from $100 \mathrm{~mm}$ to 200 mm carries a $+10.5 \%$ as $\dot{Q}_{\text {Cool }}$ percentage increment but no rising occurs for $\mathrm{L}$ increasing. The medium percentage increments on cooling power at $11 \mathrm{~m} \mathrm{~s}^{-1}$, are: $+45.4 \%$ from $100 \mathrm{~mm}$ to $200 \mathrm{~mm}$ and $+12.8 \%$ from $200 \mathrm{~mm}$ to $300 \mathrm{~mm}$.

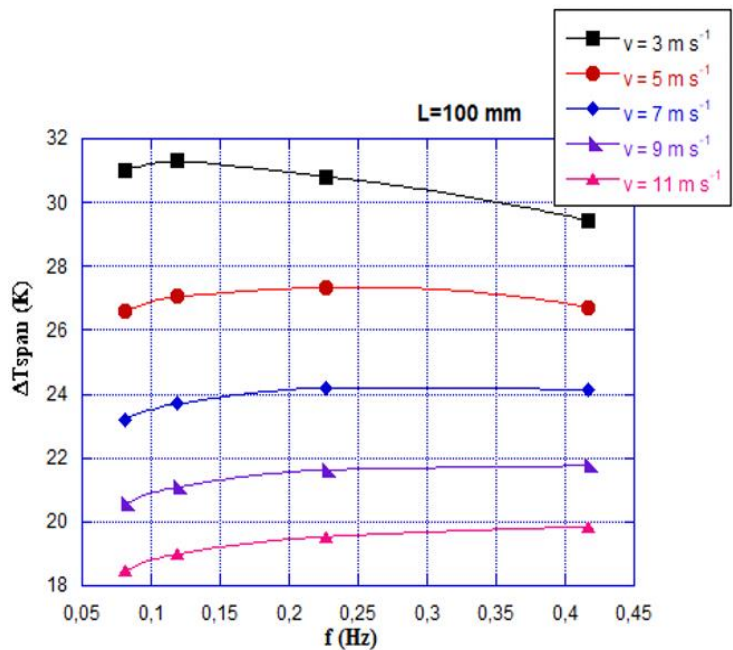

(a)

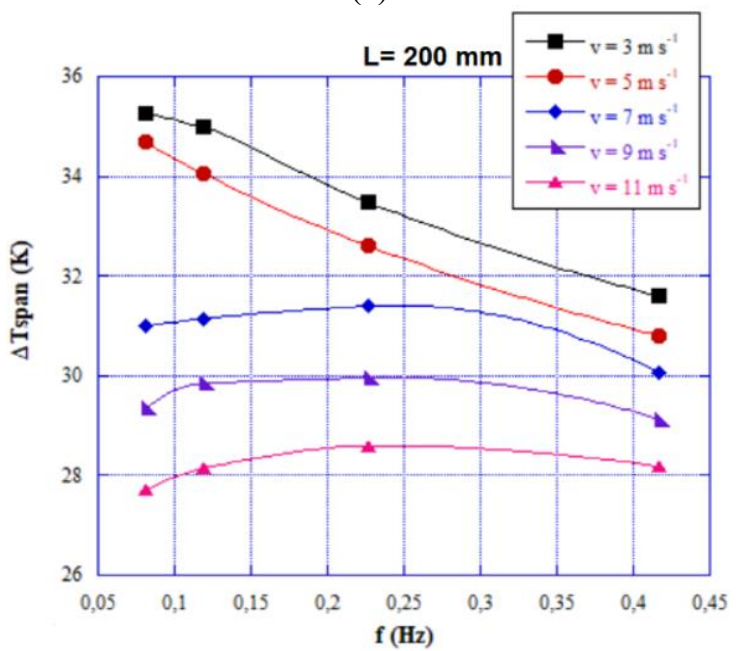

(b)

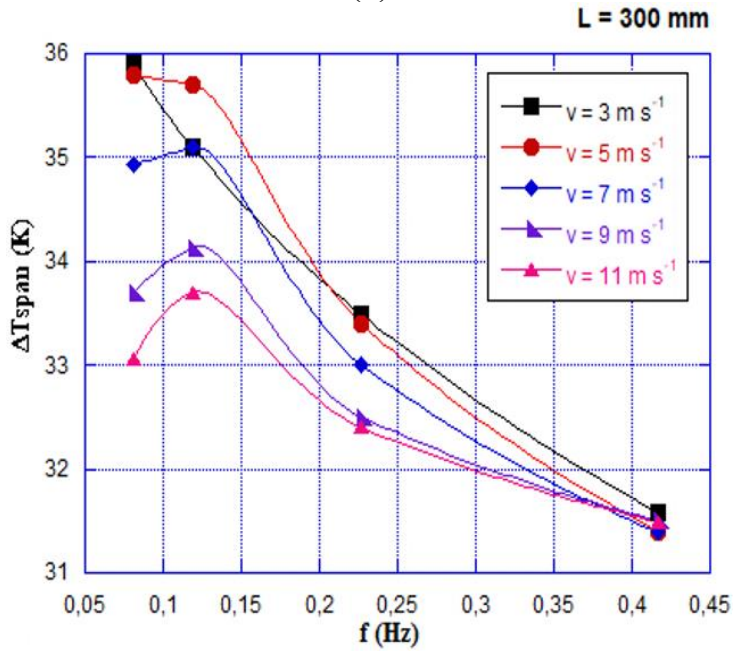

(c)

Figure 3. $\Delta \mathrm{T}_{\text {span }} \mathrm{vs} \mathrm{f}_{\mathrm{AeR}}$ parametrized for $\mathrm{v}$ when the $\mathrm{L}$ is: (a) $100 \mathrm{~mm}$; (b) $200 \mathrm{~mm}$; (c) $300 \mathrm{~mm}$ 


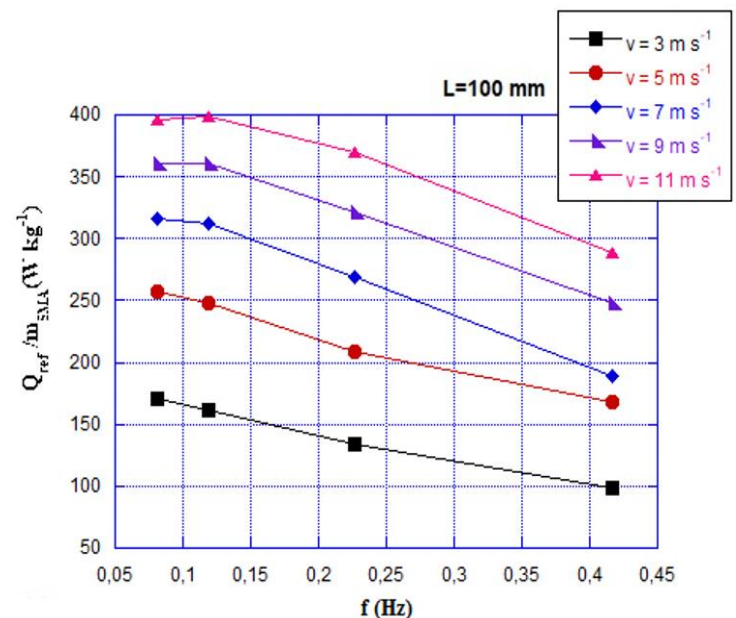

(a)

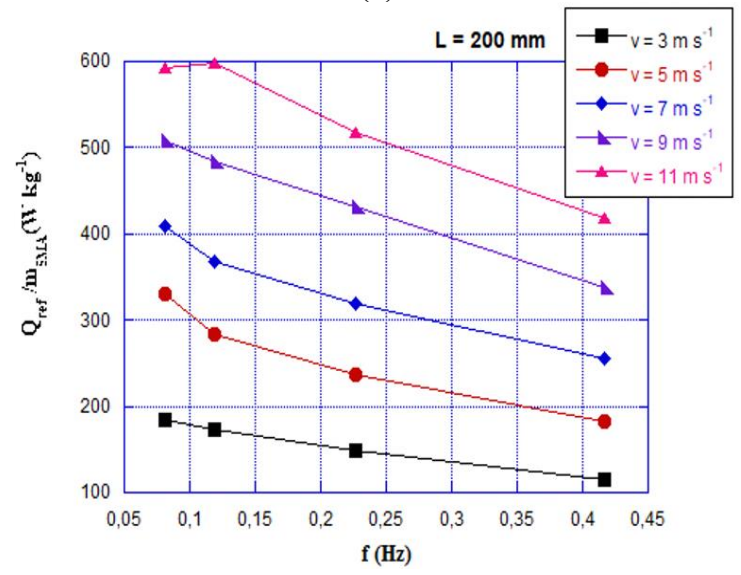

(b)

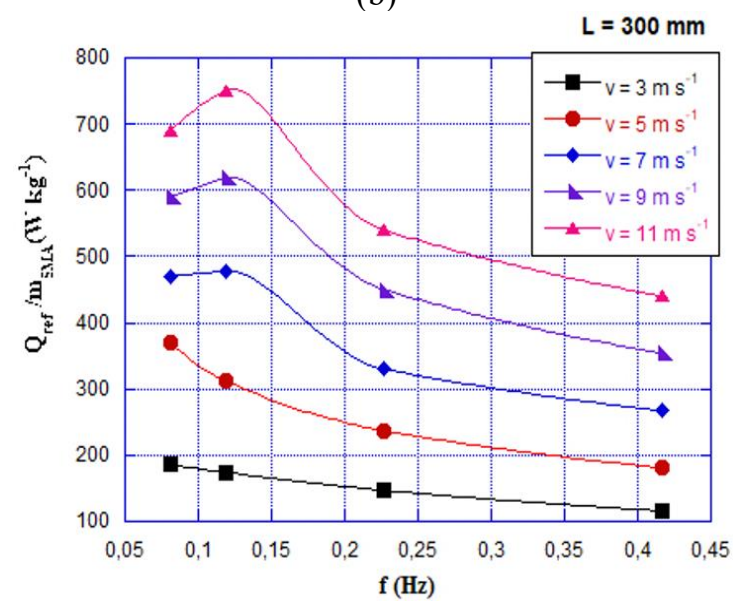

(c)

Figure 4. Cooling power per mass unit vs $\mathrm{f}_{\mathrm{AeR}}$ parametrized for $\mathrm{v}$ when $\mathrm{L}$ is: (a) $100 \mathrm{~mm}$; (b) $200 \mathrm{~mm}$; (c) $300 \mathrm{~mm}$

\section{CONCLUSIONS}

The effect of the variation of the most important operating parameters of a bunch of elastocaloric wires on the energy performances of the AeR cycle was investigated in this paper.

Specifically, the dependence of the cycle frequency and the fluid flow velocity by changing lengths and distance between the wires of a bunch of SMA wires has been studied. The bunches were designed since it will be one of several elements placed in the first Italian elastocaloric prototype. In SUSSTAIN-EL the bunches will be mounted circularly, and they will be crossed by a heat transfer fluid. The purpose is to optimize the heat exchange between the air flow and $\mathrm{Ni}-\mathrm{Ti}$ wires, so to me the prototypes showing optimized energy performances in terms of temperature span and the cooling power. The results have been presented in the paper and widely discussed; basing on this one can draw the following conclusions:

- for all the studied wire lengths the $\Delta \mathrm{T}_{\text {span }}$ reduces its value when the fluid velocity grows up on every frequency tested;

- for every fluid flow speed, the maximum value of $\Delta \mathrm{T}_{\mathrm{span}}$ is obtained at different frequency value. Fixing the velocity, the optimal frequency reduces its value if the SMA element is longer and if the distance between the wires is reduced;

- a smaller distance between two wires can provide larger $\Delta \mathrm{T}_{\text {span }}(+50 \%$ as medium increment evaluated for $\mathrm{d}=0.5$ $\mathrm{mm}$ with respect to $\mathrm{d}=1.0 \mathrm{~mm}$ );

- for every $\mathrm{v}$ and $\mathrm{f}_{\mathrm{AeR}}$, a length increase from $100 \mathrm{~mm}$ to $200 \mathrm{~mm}$ positively impacts on temperature span. There is a greater effect when the length grows on $\Delta \mathrm{T}_{\text {span }}$ from 200 $\mathrm{mm}$ to $300 \mathrm{~mm}$ with the increase of the speed, while on the contrary, there is not for $3 \mathrm{~m} \mathrm{~s}^{-1}$.

- To faster air flows are coupled larger cooling powers. This derives in the existence of a trade-off in the possibility of maximizing $\Delta \mathrm{T}_{\text {span }}$ and cooling power.

- $\quad$ Fixing both $f$ and flow speed the cooling power augments if the distance between two wires is larger.

- The $\dot{Q}_{\text {Cool }}$ per mass unit grows also for longer wires. In particular, it always grows passing from values of $\mathrm{L}$ of $100 \mathrm{~mm}$ to $200 \mathrm{~mm}$, for all the fluid speed and the frequencies tested. Whereas, passing from values of $\mathrm{L}$ of $200 \mathrm{~mm}$ to $300 \mathrm{~m}$, there is a saturation that manifests for every $\mathrm{f}_{\mathrm{AeR}}$ if the velocity is $3 \mathrm{~m} \mathrm{~s}^{-1}$. As speed increases, the saturation effect is evident only from the frequency 0.227 $\mathrm{Hz}$ upwards.

In conclusion, with respect to the cycle frequency and the air flow velocity, given the revealed trade-off between the maximization of $\Delta \mathrm{T}_{\text {span }}$ and $\dot{Q}_{\text {Cool }}$, in our opinion the most suitable solution would be to operate with values located in the centre of the tested ranges: i) $0.119 \mathrm{~Hz}$ as $\mathrm{f}_{\mathrm{AeR}}$; ii) $7 \mathrm{~m} \mathrm{~s}^{-1}$ as v; iii) $1.0 \mathrm{~mm}$ as optimal wire distance; iv) $200 \mathrm{~mm}$ as wire length.

\section{ACKNOWLEDGMENT}

This research was funded through the project "SUSSTAINEBLE" - FISR2019 04798 granted by FISR Fondo Integrativo Speciale per la Ricerca (Italian special supplementary fund for research).

\section{REFERENCES}

[1] Heath, E.A. (2017). Amendment to the Montreal protocol on substances that deplete the ozone layer (Kigali amendment). International Legal Materials, 56(1): 193-205. https://doi.org/10.1017/ilm.2016.2

[2] Qian, S., Nasuta, D., Rhoads, A., Wang, Y., Geng, Y., Hwang, Y. (2016). Not-in-kind cooling technologies: A quantitative comparison of refrigerants and system performance. International Journal of Refrigeration, 62: 177-192. https://doi.org/10.1016/j.ijrefrig.2015.10.019

[3] Barbato, M., Cirillo, L., Menditto, L., Moretti, R., Nardini, S. (2017). Geothermal energy application in 
Campi Flegrei Area: The case study of a swimming pool building. International Journal of Heat and Technology, 35(1):

S102-S107.

https://doi.org/10.18280/ijht.35Sp0114

[4] Cirillo, L., Della Corte, A., Nardini, S. (2016). Feasibility study of solar cooling thermally driven system configurations for an office building in Mediterranean area. International Journal of Heat and Technology, 34(2): 472-480. https://doi.org/10.18280/ijht.34S240

[5] Kitanovski, A., Plaznik, U., Tomc, U., Poredoš, A. (2015). Present and future caloric refrigeration and heatpump technologies. International Journal of Refrigeration, $\quad 57$ : 288-298. https://doi.org/10.1016/j.ijrefrig.2015.06.008

[6] Moya, X., Kar-Narayan, S., Mathur, N.D. (2014). Caloric materials near ferroic phase transitions. Nature Materials, 13(5):

$439-450$

https://doi.org/10.1038/nmat3951

[7] Greco, A., Vanoli, G.P. (2006). Experimental two-phase pressure gradients during evaporation of pure and mixed refrigerants in a smooth horizontal tube. Comparison with correlations. Heat and Mass Transfer, 42(8): 709725. https://doi.org/10.1007/s00231-005-0020-7

[8] Greco, A., Vanoli, G.P. (2005). Flow boiling heat transfer with HFC mixtures in a smooth horizontal tube. Part II: Assessment of predictive methods. Experimental Thermal and Fluid Science, 29(2): 199-208. https://doi.org/10.1016/j.expthermflusci.2004.03.004

[9] Greco, A., Mastrullo, R., Palombo, A. (1997). R407C as an alternative to R22 in vapour compression plant: An experimental study. International Journal of Energy Research, 21(12): 1087-1098. https://doi.org/10.1002/(SICI)1099114X(19971010)21:12<1087::AID-ER330>3.0.CO;2-Y

[10] Aprea, C., Greco, A., Maiorino, A., Masselli, C. (2020). The employment of caloric-effect materials for solidstate heat pumping. International Journal of Refrigeration, 109: 1-11. https://doi.org/10.1016/j.ijrefrig.2019.09.011

[11] Aprea, C., Cardillo, G., Greco, A., Maiorino, A., Masselli, C. (2015). A comparison between experimental and 2D numerical results of a packed-bed active magnetic regenerator. Applied Thermal Engineering, 90: 376-383. https://doi.org/10.1016/j.applthermaleng.2015.07.020

[12] Aprea, C., Greco, A., Maiorino, A. (2013). The use of the first and of the second order phase magnetic transition alloys for an AMR refrigerator at room temperature: a numerical analysis of the energy performances. Energy Conversion and Management, 70: 40-55. https://doi.org/10.1016/j.enconman.2013.02.006

[13] Aprea, C., Greco, A., Maiorino, A., Masselli, C. (2016). A comparison between different materials in an active electrocaloric regenerative cycle with a $2 \mathrm{D}$ numerical model. International Journal of Refrigeration, 69: 369382. https://doi.org/10.1016/j.ijrefrig.2016.06.016

[14] Qian, S., Geng, Y., Wang, Y., Ling, J., Hwang, Y., Radermacher, R. (2016). A review of elastocaloric cooling: Materials, cycles and system integrations. International Journal of Refrigeration, 64: 1-19. https://doi.org/10.1016/j.ijrefrig.2015.12.001

[15] Aprea, C., Greco, A., Maiorino, A., Masselli, C. (2020). The use of barocaloric effect for energy saving in a domestic refrigerator with ethylene-glycol based nanofluids: A numerical analysis and a comparison with a vapor compression cooler. Energy, 190: 116404. https://doi.org/10.1016/j.energy.2019.116404

[16] Lloveras, P., Tamarit, J.L. (2021). Advances and obstacles in pressure-driven solid-state cooling: A review of barocaloric materials. MRS Energy \& Sustainability, 8: 3-15. https://doi.org/10.1557/s43581-020-00002-4

[17] Yu, B.F., Gao, Q., Zhang, B., Meng, X.Z., Chen, Z. (2003). Review on research of room temperature magnetic refrigeration. International Journal of Refrigeration, 26(6): 622-636. https://doi.org/10.1016/S0140-7007(03)00048-3

[18] Greco, A., Aprea, C., Maiorino, A., Masselli, C. (2019). A review of the state of the art of solid-state caloric cooling processes at room-temperature before 2019 . International Journal of Refrigeration, 106: 66-88. https://doi.org/10.1016/j.ijrefrig.2019.06.034

[19] Ossmer, H., Chluba, C., Krevet, B., Quandt, E., Rohde, M., Kohl, M. (2013). Elastocaloric cooling using shape memory alloy films. In Journal of Physics: Conference Series, 476(1): 012138.

[20] Cui, J., Wu, Y., Muehlbauer, J., Hwang, Y., Radermacher, R., Fackler, S. (2012). Demonstration of high efficiency elastocaloric cooling with large $\Delta \mathrm{T}$ using NiTi wires. Applied Physics Letters, 101(7): 073904. https://doi.org/10.1063/1.4746257

[21] Bruederlin, F., Bumke, L., Chluba, C., Ossmer, H., Quandt, E., Kohl, M. (2018). Elastocaloric cooling on the miniature scale: a review on materials and device engineering. Energy Technology, 6(8): 1588-1604. https://doi.org/10.1002/ente.201800137

[22] Zeng, K., Schmid-Fetzer, R., Huneau, B., Rogl, P., Bauer, J. (1999). The ternary system Al-Ni-Ti part II: thermodynamic assessment and experimental investigation of polythermal phase equilibria. Intermetallics, 7(12): 1347-1359. https://doi.org/10.1016/S0966-9795(99)00055-2

[23] Al Hasan, N.M., Hou, H., Sarkar, S., Thienhaus, S., Mehta, A., Ludwig, A., Takeuchi, I. (2020). Combinatorial synthesis and high-throughput characterization of microstructure and phase transformation in $\mathrm{Ni}-\mathrm{Ti}-\mathrm{Cu}-\mathrm{V}$ quaternary thin-film library. Engineering, 6(6): 637-643. https://doi.org/10.1016/j.eng.2020.05.003

[24] Mosca, H.O., Bozzolo, G., Del Grosso, M.F. (2012). Atomistic modeling of ternary additions to NiTi and quaternary additions to $\mathrm{Ni}-\mathrm{Ti}-\mathrm{Pd}, \mathrm{Ni}-\mathrm{Ti}-\mathrm{Pt}$ and $\mathrm{Ni}-\mathrm{Ti}-$ Hf shape memory alloys. Physica B: Condensed Matter, 407(16):

3244-3247. https://doi.org/10.1016/j.physb.2011.12.077

[25] Mañosa, L., Jarque-Farnos, S., Vives, E., Planes, A. (2013). Large temperature span and giant refrigerant capacity in elastocaloric $\mathrm{Cu}-\mathrm{Zn}-\mathrm{Al}$ shape memory alloys. Applied Physics Letters, 103(21): 211904. https://doi.org/10.1063/1.4832339

[26] Yuan, B., Zhu, X., Zhang, X., Qian, M. (2019). Elastocaloric effect with small hysteresis in bamboograined $\mathrm{Cu}-\mathrm{Al}-\mathrm{Mn}$ microwires. Journal of Materials Science, 54(13): 9613-9621. https://doi.org/10.1007/s10853-019-03592-8

[27] Xiao, F., Fukuda, T., Kakeshita, T., Jin, X. (2015). Elastocaloric effect by a weak first-order transformation associated with lattice softening in an Fe-31.2 Pd (at.\%) alloy. Acta Materialia, 87: 8-14. https://doi.org/10.1016/j.actamat.2015.01.004 
[28] Patel, S., Chauhan, A., Vaish, R., Thomas, P. (2016). Elastocaloric and barocaloric effects in polyvinylidene di-fluoride-based polymers. Applied Physics Letters, 108(7): 072903. https://doi.org/10.1063/1.4942000

[29] Xie, Z., Sebald, G., Guyomar, D. (2017). Comparison of elastocaloric effect of natural rubber with other caloric effects on different-scale cooling application cases. Applied Thermal Engineering, 111: 914-926. https://doi.org/10.1016/j.applthermaleng.2016.09.164

[30] Cirillo, L., Farina, A.R., Greco, A., Masselli, C. (2021). Preliminary numerical investigation on the optimization of a single bunch of elastocaloric elements to be employed in an experimental device. TECNICA ITALIANA-Italian Journal of Engineering Science, 65(2-4): 242-249. https://doi.org/10.18280/ti-ijes.652416

[31] Cirillo, L., Farina, A.R., Greco, A., Masselli, C. (2021). The optimization of the energy performances of a single bunch of elastocaloric elements to be employed in an experimental device. Thermal Science and Engineering Progress, 27:

101152 https://doi.org/10.1016/j.tsep.2021.101152

[32] Tušek, J., Engelbrecht, K., Mañosa, L., Vives, E., Pryds, N. (2016). Understanding the thermodynamic properties of the elastocaloric effect through experimentation and modelling. Shape Memory and Superelasticity, 2(4): 317-329. https://doi.org/10.1007/s40830-016-0094-8

[33] Ossmer, H., Lambrecht, F., Gültig, M., Chluba, C., Quandt, E., Kohl, M. (2014). Evolution of temperature profiles in TiNi films for elastocaloric cooling. Acta Materialia, $81:$

$9-20$ https://doi.org/10.1016/j.actamat.2014.08.006

\section{NOMENCLATURE}

\section{Roman symbols}

A area, $\mathrm{m}^{2}$

c specific heat capacity, $\mathrm{J} \mathrm{kg}^{-1} \mathrm{~K}^{-1}$

D diameter, $\mathrm{mm}$

$\mathrm{d} \quad$ Distance between two wires, $\mathrm{mm}$

f frequency, $\mathrm{Hz}$

$\mathrm{G} \quad$ elastocaloric term, $\mathrm{kJ} \mathrm{m}^{-3}$

$\mathrm{H} \quad$ Latent heat, $\mathrm{J} \mathrm{g}^{-1}$

convective heat transfer coefficient, $\mathrm{W} \mathrm{m} \mathrm{m}^{-2} \mathrm{~K}^{-1}$ thermal conductivity, $\mathrm{W} \mathrm{m}^{-1} \mathrm{~K}^{-1}$
L length of the wire, $\mathrm{mm}$

m Mass, kg

$\mathrm{m}^{\cdot} \quad$ flow rate, $\mathrm{kg} \mathrm{s}^{-1}$

n number of times

$\mathrm{p} \quad$ pressure, $\mathrm{Pa}$

Pr Prandtl number, -

Q $\quad$ power, W

$\mathrm{T}$ temperature, $\mathrm{K}$

$\mathrm{t} \quad$ time, $\mathrm{s}$

$\mathrm{u} \quad \mathrm{X}$-velocity field component, $\mathrm{m} \mathrm{s}^{-1}$

$\mathrm{V} \quad$ volume, $\mathrm{m}^{3}$

$\mathrm{v} \quad \mathrm{y}$-velocity field component, $\mathrm{m} \mathrm{s}^{-1}$

$\mathrm{v}^{-} \quad$ velocity vector, $\mathrm{m} \mathrm{s}^{-1}$

$\mathrm{w} \quad$ loading/unloading work, $\mathrm{J} \mathrm{g}^{-1}$

$\mathrm{x} \quad$ longitudinal spatial coordinate, $\mathrm{m}$

y orthogonal spatial coordinate, $\mathrm{m}$

\section{Greek symbols}

$\Delta \quad$ finite difference

$\varepsilon \quad$ strain, $\mathrm{N}$

$\theta \quad$ period of the AeR cycle, $\mathrm{s}$

$\mu \quad$ dynamic viscosity, $\mathrm{Pa} s$

$v \quad$ cinematic viscosity, $\mathrm{m}^{2} \mathrm{~s}^{-1}$

$\xi \quad$ volume fraction of the superelastic phase, -

$\rho \quad$ density, $\mathrm{kg} \mathrm{m}^{-3}$

$\sigma \quad$ uniaxial stress, $\mathrm{MPa}$

$\tau \quad$ convective heat exchange transient constant, $\mathrm{s}$

$\psi \quad$ probability,

\section{Subscripts}

A Austenitic

AM Austenite-to-Martensite transformation

ad adiabatic

air air

C cooling

env environment

f fluid

load loading

M Martensitic

MA Martensite-to-Austenite transformation

net net

SMA Shape Memory Alloy

span span

unload unloading 